\title{
Disjoint Routes for On Demand Routing Protocols in Ad Hoc Networks
}

\author{
E. Papapetrou, F.-N. Pavlidou \\ Aristotle University of Thessaloniki, School of Engineering, \\ Dept. of Electrical \& Computer Engineering, Telecommunications Division, \\ 54006 Thessaloniki, Greece \\ Tel/Fax : +30 31996285
}

\begin{abstract}
Wireless mobile networks are characterized by stochastic topology variations. Sudden movements of mobile hosts in and out of each other's range impose limitations on the system performance. Routing protocols have been proposed for producing stable routes. All these protocols address the issue of route stability rather than connection stability. This paper proposes a new protocol for enhancing stability of connections rather than routes, without increasing the related overhead. The new protocol is based on the employment of a number of disjoint paths in each route discovery phase. Its performance is evaluated by simulations in the Java programming language that present adaptability to increased mobility.
\end{abstract}

\section{INTRODUCTION}

Recent technology advances have increased the penetration of portable laptops and handheld devices around the world resulting to a continuously increasing demand for connectivity. A new kind of wireless computer networks has thus appeared that operate without the need of a fixed infrastructure. These networks, known as ad hoc networks, can be easily and quickly deployed. As they consist of mobile hosts within a relatively small communication range, they are prone to link failures when mobile hosts move randomly in or out of each other's range. Thus, connectivity in ad hoc networks varies stochastically. This fact deeply affects the choice of an appropriate routing technique.

Traditional table-driven routing protocols [1] cannot perform efficiently in such an environment [2]. On-demand routing protocols [1] have been proposed as an effective solution to the problem. Most of them are based on the source routing technique known from IEEE 802 LANs implementation. Routes are discovered only when needed using a route discovery procedure. This means that either a user request or a route break down may cause a new route discovery. In this way overhead packets are minimized since only a number of topology changes is considered. The most representative protocol of source routing is the Dynamic Source Routing (DSR) protocol [3]. But although DSR outperforms table driven protocols, link failures still degrade the network performance, especially when the hosts' mobility increases. The performance degradation concerns the packet delivery ratio and the delay jitter imposed to data packets.
Many modifications regarding the route discovery phase of the DSR protocol have been proposed in the literature [1]. Most of them are concerned with the introduction of new metrics for choosing the source routes. The most desired feature of a routing protocol for ad hoc networks is its ability to setup fairly stable routes. Two protocols have been proposed as adequate for this target, the Signal Stability Adaptive (SSA) protocol [4] and the Route-Lifetime Assessment based Routing (RABR) protocol [5].

In SSA each host classifies its neighbors as strongly or weakly connected. In each route discovery phase, a host propagates the request packet only if it comes from a strongly connected host. Setting the threshold for considering a neighboring node strongly or weakly connected is not a dynamic procedure. Therefore, depending on the network status, SSA may fail to discover stable routes. In this case, route discovery packets are propagated over weak links but high delay is imposed since a host waits for an amount of time before allowing this transmission. Additionally, paths that are not considered stable in SSA may be optimal in terms of end-to-end delay [4].

RABR is based on DSR. It introduces a special metric to evaluate and assess the stability of a link. Although it achieves improvement in terms of throughput, as a side effect it introduces routing overhead. This is because the proposed metric follows an additive rule, [6] so hosts must propagate all the request packets they receive. Let us consider the route discovery phase in Fig. 1. taking transmission delay (Fig. 1a) as the stability metric. Then if a node encounters multiple request packets for the same route, it transmits only the first one and ignores all the others. Thus, transmissions from hosts stop at time $t_{3}$ (Fig. 1a) and although hosts 2 and 3 receive two copies of the request packet then propagate only one. On the contrary if the used metric follows an additive rule each node must forward all the copies of the request packet because all the possible combinations must be considered. Thus hosts 2 and 3 in Fig. 1b must transmit all the copies of the route request packet. This leads to the increase of the protocol overhead, and furthermore to the increase of the endto-end delay since the destination node must reply to the originator after the collection of all the packets. 

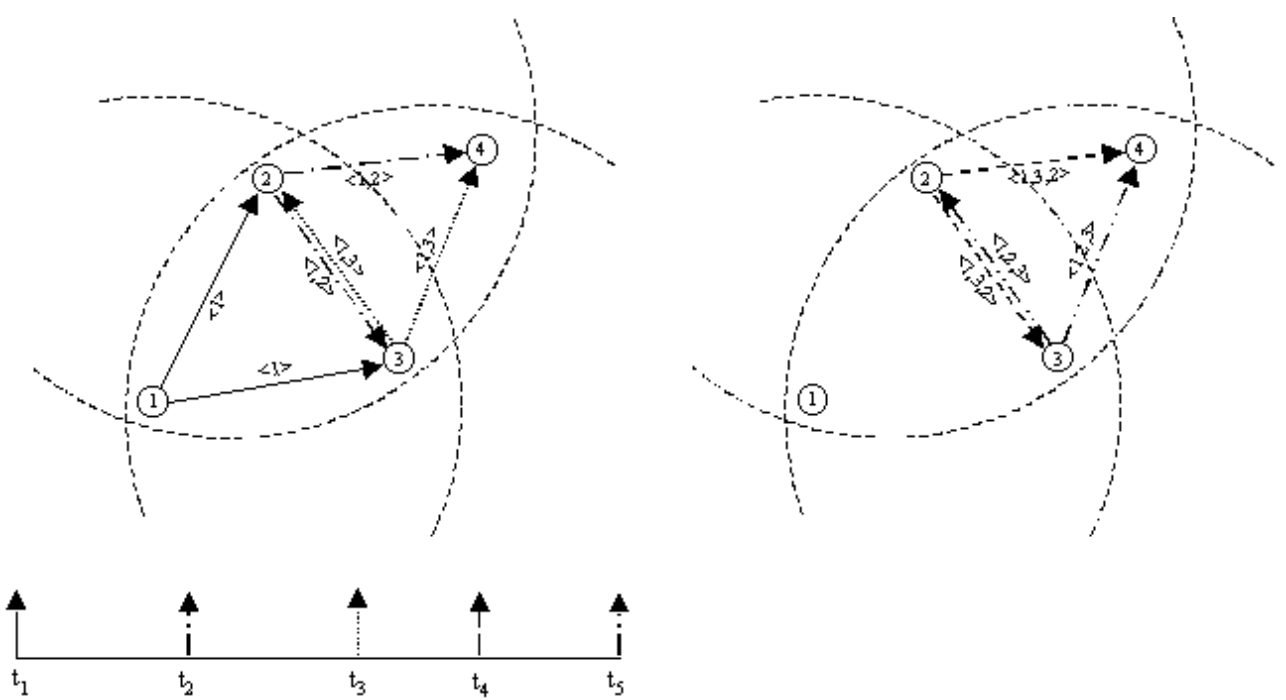

Figure 1. a) Example of route discovery when the used metric is the transmission delay, b) additional transmissions needed when the metric follows an additive rule.

In this framework a new methodology to achieve "stability" must be developed. In general, stability must be achieved for origin/destination connections rather than routes. This means that although a route may break down, a second «stand by» route may enhance the connection stability. This route will substitute immediately the broken one so that delay jitter and dropped packets are minimized. The availability of a "stand by" route and its correlation to the default path are crucial issues for the connection "stability". To this point we shall present in the following a new protocol based on the use of disjoint routes for enhancing the stability in a connection basis. The positive result is a quite improved protocol performance.

The rest of the paper is structured as follows. In Section II the new protocol is presented in detail. Then, in Section III we discuss the simulation framework and the implementation for evaluating the new protocol. In Section IV the results of our simulation study are presented, leading to useful conclusions in Section V where topics for future work are also addressed.

\section{DISJOINT PATHS ROUTING PROTOCOL}

The proposed protocol is based on the DSR technique with modifications regarding the route discovery and maintenance phases as described in the following in detail.

\section{A. Route Discovery}

The first modification of the DSR protocol is made in its route discovery phase, so that the destination host be able for multiple responses to a route request. In this way more than one paths are formed. Of course the routing overhead is increased since more reply packets must be transmitted from the destination back to the originator of the route request. But on the other hand using more than one route leads to a reduction of the number of route requests. Consider the case that a route is disabled. If a second path is available, it is not necessary to initiate a new route discovery procedure. The simulation results show that for a relatively small number of alternative routes the increase in overhead load can be insignificant.

Permitting the destination host to respond with more than one route can be advantageous only if during the route discovery phase each intermediate host propagates only the first copy of a route request packet (i.e. the formed paths are disjoint). This suppresses routing overhead but also improves the protocol performance in the following manner. The stability of a connection between two hosts depends greatly on the discovered paths. In our implementation all the discovered routes are disjoint since there is no way that two copies of the same request packet arriving at the destination host, have encountered the same intermediate host, a constraint posed by the basic DSR protocol. This would require that this intermediate host propagated two copies of the same request packet. Using disjoint routes increases the probability that when the default route of a connection is disabled another route will be available, therefore avoiding the launch of another route discovery phase.

\section{B. Route Maintenance}

The identification of broken links in the route maintenance mechanism of our protocol is the same as in the DSR protocol. This means that if a transmitting host times out (after retransmitting the packet for a number of times), it then sends an error packet to the originator of the original packet. Upon receiving an error packet, a host instead of initiating a route request searches its cache for another route to the same destination. The possibility of finding a new route in its cache increases with the number of routes that are discovered in 
each route request. Furthermore, using disjoint paths ensures us that a route error packet will not delete more than one cached route. In this way the number of route discoveries is suppressed, leading to the decrease of the routing overhead.

\section{SIMULATION FRAMEWORK}

The code for implementing the new protocol, was developed in the Java programming language. At the link layer we simulated also the discrete coordination function (DCF) of the IEEE 802.11 Wireless LAN standard [7]. A code for the DSR protocol was also developed in the same way. The first set of simulations devoted to test the compatibility of the new simulator giving exactly the results obtained by the network simulator $n s$ of the Lawrence Berkeley National laboratory.

\section{A. Simulation Scenarios}

To evaluate the new protocol we simulated a scenario of 100 nodes moving randomly in an area of $670 \times 670 \mathrm{~m}^{2}$. The nominal communication range of each host was considered equal to $250 \mathrm{~m}$. The simulations have been run for $600 \mathrm{secs}$ of simulation time.

In order to explore the effect of using more than two disjoint routes we have tested different scenarios where in each route request the protocol discovers 2,3 or 5 routes.

\section{B. Data Transmission Model}

We simulated 50 connections each one emerging from a different host. Packets for each connection are generated exponentially. The packet generation rate of each host was set to $1 \mathrm{packet} / \mathrm{sec}$. The channel capacity was set to $2 \mathrm{Mbits} / \mathrm{s}$, while the packet size is 512 bytes for data packets and 192 bits for protocol packets. Each data packet can be buffered for a maximum time of $250 \mathrm{msecs}$. If this time expires then the packet is dropped.

\section{Mobility Pattern}

The hosts in the simulations move according to the Random Waypoint algorithm [2]. Each host chooses its destination location and moves towards that destination with a velocity $v$. On reaching the destination, the host pauses for a time interval and then chooses another location. The speed of each node is uniformly chosen in the range of 0 to $v_{\max }$ $\mathrm{m} / \mathrm{sec}$.

The simulations are run for values of the pause time equal to $0,100,300$ and $600 \mathrm{secs}$. Furthermore, we tested different values of $v_{\max }(0,5,10,15$ and $20 \mathrm{~m} / \mathrm{sec})$.

\section{Simulation Results}

The performance behavior of the new protocol was evaluated through two different experiments. In the first experiment we conducted simulations for different pause times using a maximum node speed of $20 \mathrm{~m} / \mathrm{sec}$. We performed different sets of simulations altering the number of discovered paths in each route discovery phase of our protocol. In the second experiment the number of discovered paths is set to 2 and the protocol is tested through a range of maximum node speed values.

In Fig. 2 to 5 the relevant performance of the proposed protocol and the DSR protocol is depicted with respect to the hosts' pause time. As can been seen in Fig. 2 the proposed protocol outperforms DSR in terms of overhead packets created. Contrary to DSR, the new protocol does not initiate a route discovery phase each time a route is broken but it uses the existent (disjoint) route to continue the transmission of packets. As a result the overhead is reduced even though the number of reply packets is increased relevant to the DSR case. A confirmation of the reduced number of route discoveries in the new protocol is given in Fig. 3 where the total number of route discoveries is presented.

This advantage of the new protocol is obscured when more than two routes are used. In this case the number of reply packets grows. On the other hand the impact of additional routes in the reduction of the number of route discoveries reduces. Consequently the rate of improvement relative to DSR is reduced.

In Fig. 4 the percentage of delivered packets is depicted. The proposed protocol slightly improves the performance of DSR when two paths are used. In the other cases where more paths are employed the performance degrades as a result of the fact that the percentage of the stale routing information is becoming larger. Considering a constant rate of route failure the greater the number of discovered paths per route discovery is, the less these paths are used. Thus, it is more possible for these paths to be broken when a host tries to utilize them.

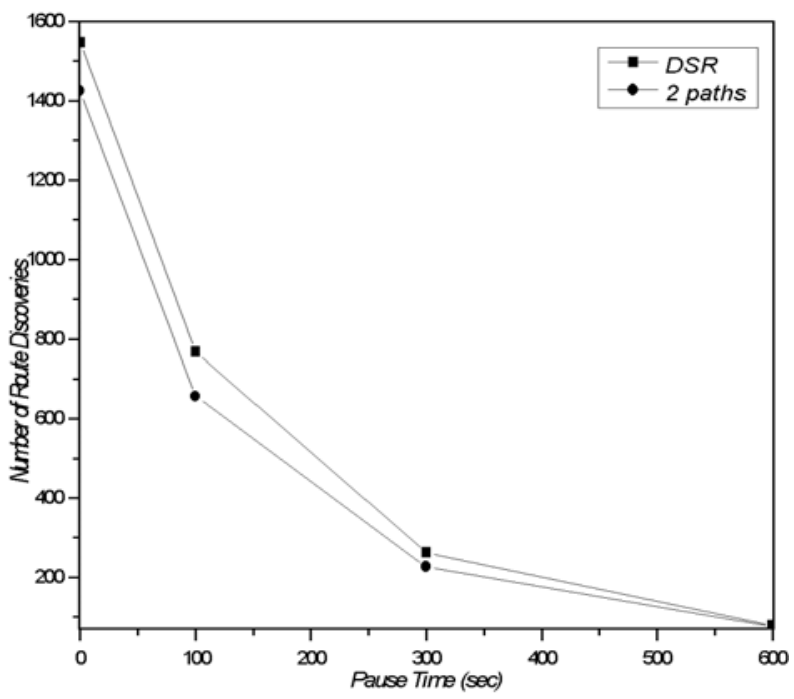

Figure 2. Routing overhead vs. pause time for DSR and when 2,3 or 5 disjoint routes are used. 


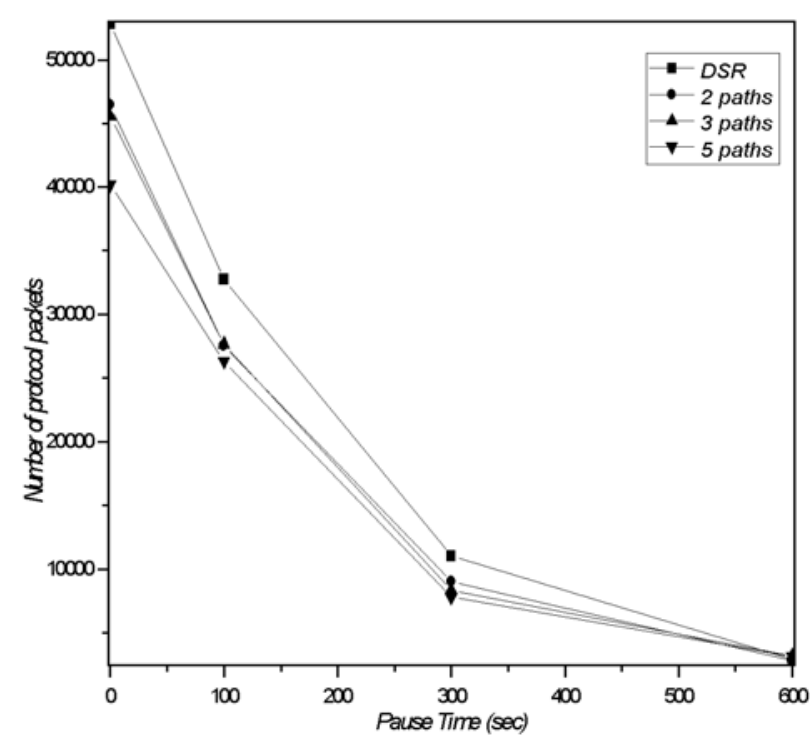

Figure 3. Total number of route discoveries vs. pause time for DSR and when 2 disjoint routes are used.

Beyond the availability of disjoint paths another important issue is their quality in terms of the included hops. In Fig. 5 the mean number of hops the serviced packets encountered is depicted. It is observed that the new protocol is superior in any case. The reason for this is the feature of DSR called salvaging [3]. Consider the case that a data packet is forwarded along its source. If a host in this route finds out that the next hop is unreachable except from sending back to the originator of the request a route error packet, also tries to divert the packet through a new route to the destination if it finds one in its case. This "salvaging " procedure results to the increase of the mean number of hops. In the modified protocol these cases are more rare keeping the mean number of hops low.

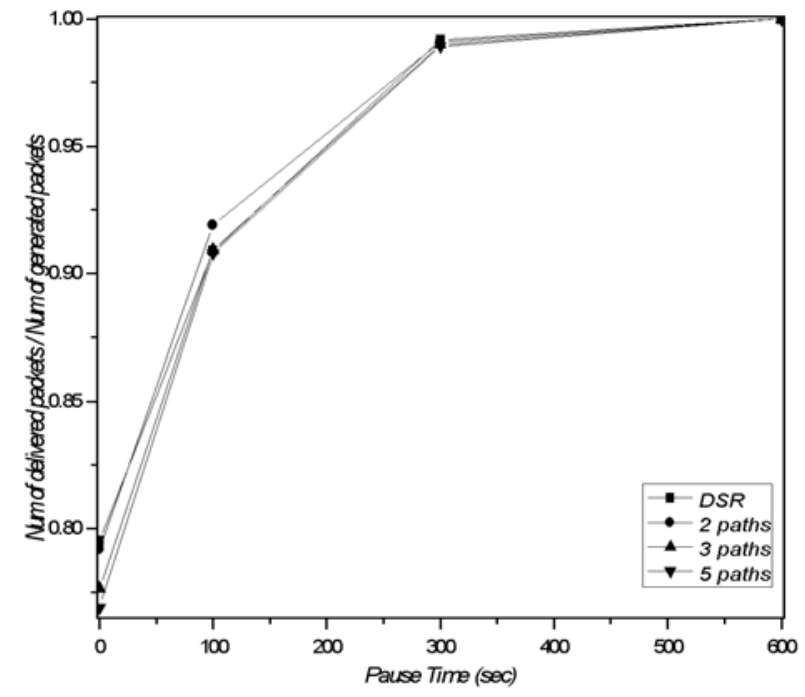

Figure 4. Serviced packets ratio vs. pause time for DSR and when 2,3 or 5 disjoint routes are used.

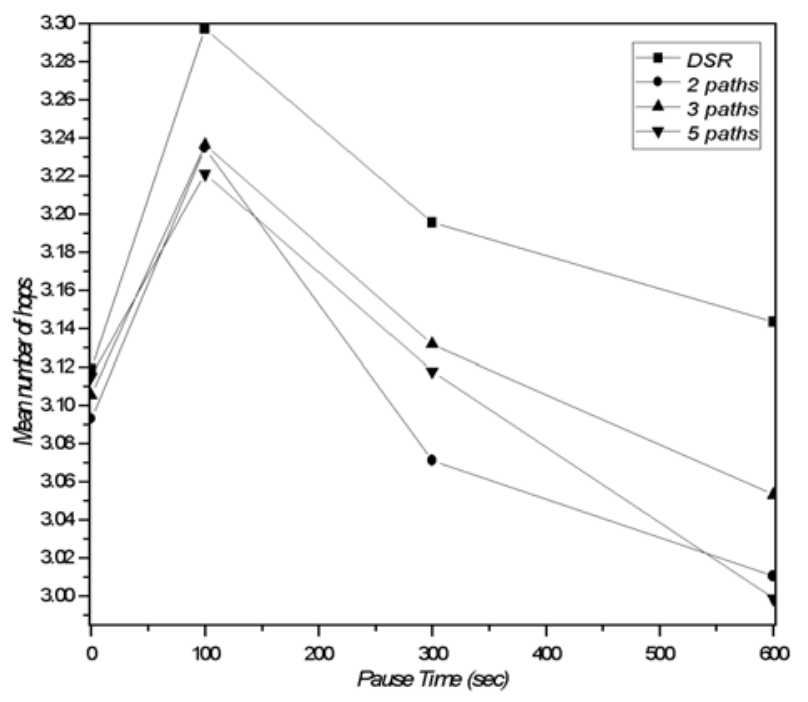

Figure 5. Mean number of hops vs. pause time for DSR and when 2,3 or 5 disjoint routes are used.

An important quality criterion for a routing protocol for ad hoc networks is the time needed to discover a path. Especially in cases where the transferred data are delay sensitive small discovery times reduce the imposed delay jitter. In Fig. 6 the distribution of the route discovery times is presented for the two protocols.

As can be observed the discovery time for our protocol is shifted to lower values. It must be noticed that the cases that our protocol replaces a broken route with an existent disjoint route (therefore the delay can be considered equal to 0) have been excluded from this graph. But still the performance of the new protocol is superior. This is caused by the fact that the cache memories of all hosts are enhanced with more paths, enabling them to reply to route requests in more cases.

After evaluating the performance of the new protocol using each time a different number of discovered routes (per

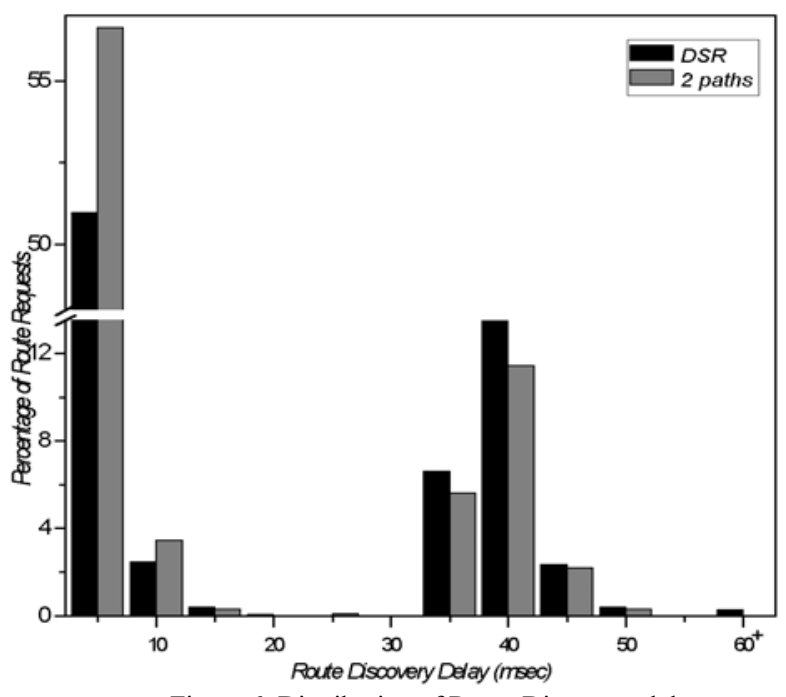

Figure 6. Distribution of Route Discovery delays. 


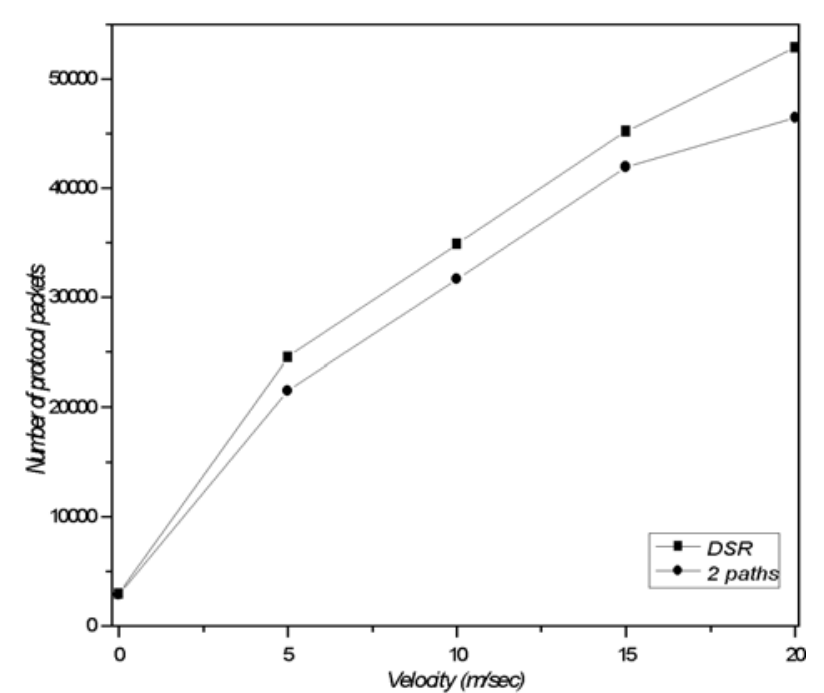

Figure 7. Routing overhead vs. mobility for DSR and when 2 disjoint routes are used.

route discovery phase) we decided to investigate its performance through a wide mobility range. Thus, we executed a set of simulations changing in each simulation the mean hosts' speed from $0 \mathrm{~m} / \mathrm{sec}$ to $20 \mathrm{~m} / \mathrm{sec}$. In each simulation the pause time was considered equal to $0 \mathrm{sec}$ and we set the number of discovered paths per route discovery equal to two for the new protocol.

In Fig. 7 the superiority of the new protocol with respect to DSR is illustrated across the whole mobility range. It can be observed that the difference of the two protocols is intensified as the mean velocity increases. This fact renders the new protocol appropriate for networks that are characterized by high mobility. The tradeoff is the small decrease in the ratio of delivered to generated packets, that is illustrated in Fig. 8.

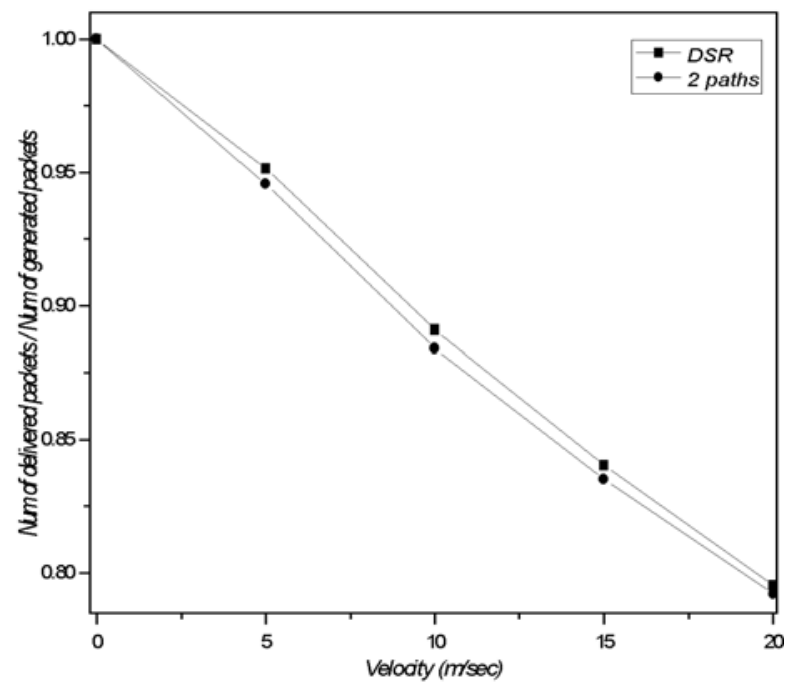

Figure 8. Serviced packets ratio vs. mobility for DSR and when 2 disjoint routes are used.

As can be seen this decrease is less than $1 \%$. More important is the fact that the curves of the two protocols are stable. This means that the difference is not increasing with respect to mean hosts' speed. It is the authors' opinion and an issue for further research that this difference is due to the small delay limit that was considered acceptable in our simulations (250 msecs).

\section{CONCLUSIONS AND FUtURE WORK}

In this paper the utilization of disjoint routes for on demand routing protocols for ad hoc networks has been investigated. The new methodology proved to have an advantage over the well-known DSR protocol in terms of produced overhead, route quality and route discovery time while keeping the complexity low. Although the proposed modifications were tested in DSR, it is clear that any other on demand protocol that operates on the basis of route discovery and maintenance mechanisms is a suitable choice. Investigating their performance is an area of future work but we soundly believe that the modifications impact will be the same or better as the underlying mechanism is common for all these protocols.

\section{REFERENCES}

[1] Elizabeth M. Royer, Chai-Keong Toh, "A Review of Current Routing Protocols for Ad Hoc Mobile Wireless Networks", IEEE Personal Communications, Vol. 6, No. 2, pp. 46-55, April 1999.

[2] Josh Brosh, D.A. Maltz., D.B. Johnson, Y.C. Hu and J. Jetcheva, "A Performance Comparison of Multi-Hop Wireless Ad Hoc Network Routing Protocols" in Proceedings of the Fourth Annual ACM/IEEE International Conference on Mobile Computing and Networking (MobiCom'98), October 25-30, 1998, Dallas, Texas, USA.

[3] David B. Johnson, Davis A. Maltz, "The Dynamic Source Routing Protocol for Mobile Ad Hoc Networks" October 1999 IETF Draft, 49 pages. http://www.ietf.org/internet-drafts/draft-ietf-manet-dsr03.txt

[4] R. Dube, C.R. Rais, K.Y. Wang and S. K. Tripathi, "Signal Stability based adaptive routing for Ad Hoc Mobile networks", IEEE Pers. Comm., Feb. 1997, pp. 36-45.

[5] S. Agarwal, A. Ahuja, J, P. Singh and R. Shorey, "Route-Lifetime Assessment Based Routing (RABR) protocol for mobile ad-hoc networks", in Proc. ICC 2000, pp. 1697-1701.

[6] Z. Wang and J. Crowcroft, "Quality-of-service routing for supporting multimedia applications", IEEE JSAC Vol. 14, No. 7, Sept. 1996, pp. 1228-1234.

[7] IEEE Computer Society LAN MAN Standards Committee. Wireless LAN Medium Access Control $(M A C)$ and Physical Layer (PHY) Specifications, IEEE Std 802.11-1997. The Institute of Electrical and Electronics Engineers, New York, New York, 1997. 